\title{
Spontaneous Retinal Pigment Epithelium Rips under Fovea with Preserved Good Visual Acuity Outcome
}

\author{
Sunny Chi Lik Au* and Callie Ka Li Ko \\ Department of Ophthalmology, Tung Wah Eastern Hospital, Hong Kong, China
}

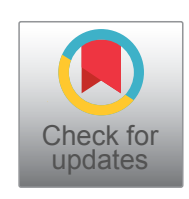

\begin{abstract}
Keywords
Retinal pigment epithelium, Photoreceptor cells, Optical coherence tomography, Fluorescein angiography, Macular degeneration (MeSH terms)
\end{abstract}

\section{Manuscript}

An 80-year-old lady was follow up in retina clinic for right only eye with Pigment Epithelium Detachment (PED). She had a history of left eye occult choroidal neovascularization, developed macula scar and legal blindness years ago despite repeated anti-Vascular Endothelial Growth Factor (antiVEGF) injection. Upon regular follow-up, her right eye macula developed serous PED centered at fovea, enlarging with time (Figure 1A, Figure 1B, Figure $1 \mathrm{C}$ and Figure 1D) throughout 2-years. There was no leakage, nor polyps/abnormal branch vascular network all along on serial Fundus Fluorescein Angiography (FFA) and Indocyanine Green Angiography respectively, thus anti-VEGF injection was never given to her right eye, or photodynamic therapy.

With conservative treatment of observation, her right eye PED developed grade 3 Retinal Pigment Epithelium (RPE) rip spontaneously [1], 2-years since the appearance of PED with progressive enlargement. The RPE rip edge retracted just underneath the fovea, with retained photoreceptor layers. (Figure 1F) Subretinal fluid (SRF) beneath the rip appeared as pooling, but no leakage on FFA. (Figure 2) The RPE rip together with the SRF morphology remained static throughout the subsequent 8-month of follow up, (Figure 3) without any further retraction or RPE regeneration over the rip edge. Her visual acuity remains at Snellen 6/9 throughout before and up to 8 months after the spontaneous rip on latest follow up.

RPE rips were common after anti-VEGF injections, and generally caused poor visual acuity [2], with exceptions depending on morphology and location of the rip. Although studies on risk factors for RPE rip mainly focused on anti-VEGF injection cases, when applying the rationale of increasing mechanical stress from rising hydrostatic pressure on our case of spontaneous RPE rip, height $>600 \mu \mathrm{m}$ and basal diameter $>$ $500 \mu \mathrm{m}$ were deemed high risk [3], even though it was not of rapid progression in a short period of time (2-years).

Visual acuity determinant after an RPE rip mainly lies on the viability of photoreceptors. Hypotheses on good vision after RPE tear suggested that photoreceptors did not need an intact RPE to survive for up to 325 days [4]. Repair mechanisms of RPE, migration and resurfacing the denuded area, RPE-independent visual cycle for cone photopigment are all hypotheses for good visual outcome despite foveal location of RPE rip. Concerning outcomes of Age-Related Macular Degeneration, other than exudation or Geographic Atrophy, it could be atypical RPE defect with preservation of photoreceptor cells [5]. Spontaneous rip is not common, and factors driving the post-rip morphology for preservation of good vision, as in our case, are still uncertain. With the gaining knowledge on photoreceptors survival with RPE rip, retinal surgeons whether should just observe the enlarging PED or surgically manipulate it for better visual outcomes to prevent blindness warrant future investigation.

*Corresponding author: Sunny Chi Lik Au, MRCS, Department of Ophthalmology, MO office, 9/F, Lo Ka Chow Memorial Ophthalmic Centre, Tung Wah Eastern Hospital, 19 Eastern Hospital Road, Causeway Bay, Hong Kong, China, Tel: (852)-2595-7031

Accepted: October 09, 2020

Published online: October 11, 2020

Citation: Sunny CLA, Callie KLK (2020) Spontaneous Retinal Pigment Epithelium Rips under Fovea with Preserved Good Visual Acuity Outcome. J Healthcare 3(1):33-37 

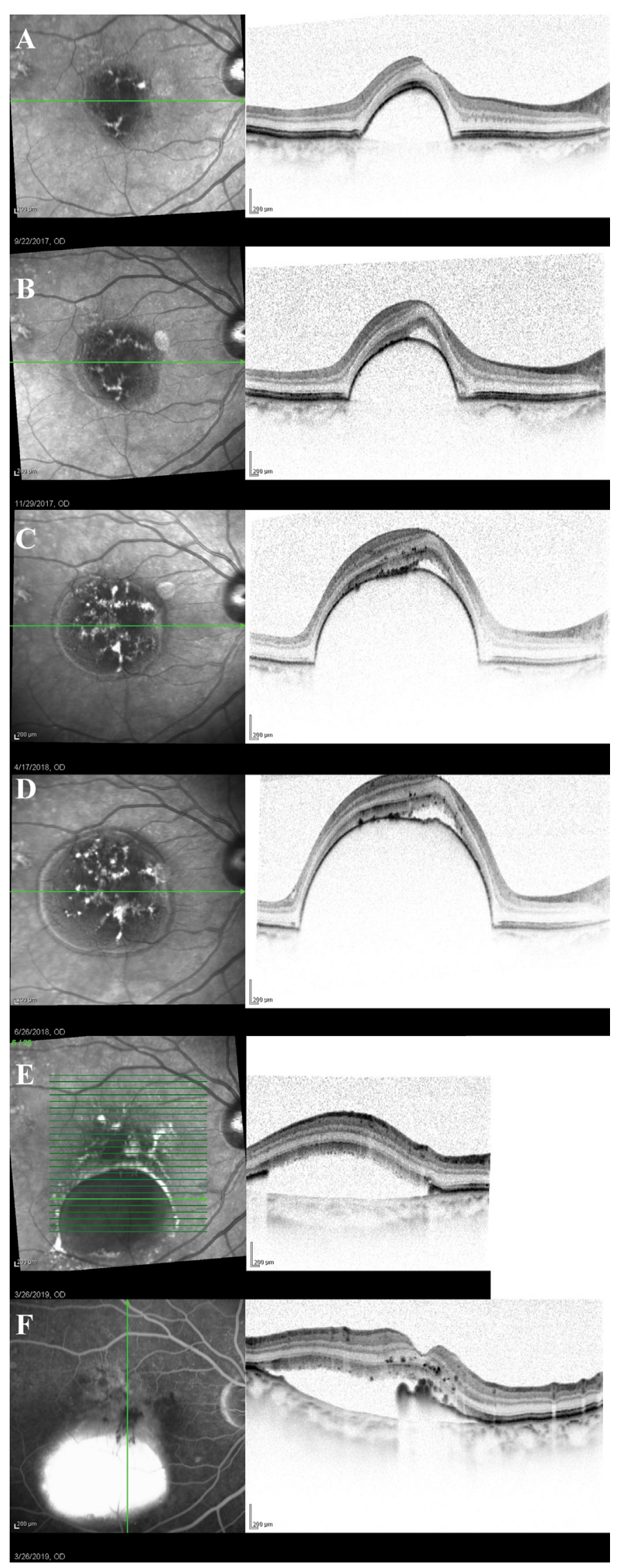

Figure 1: Spontaneous retinal pigment epithelium rips under fovea with preserved good visual acuity outcome.

Serial optical coherence tomography of the right eye macula showing the morphological changes of the enlarging pigment epithelium detachment at fovea from 22/9/2017-26/3/2019. (1A to 1D) Retinal pigment epithelium waviness appeared (Figure 1D), before rip developed spontaneously (Figure 1F). 
Citation: Sunny CLA, Callie KLK (2020) Spontaneous Retinal Pigment Epithelium Rips under Fovea with Preserved Good Visual Acuity Outcome. J Healthcare 3(1):33-37

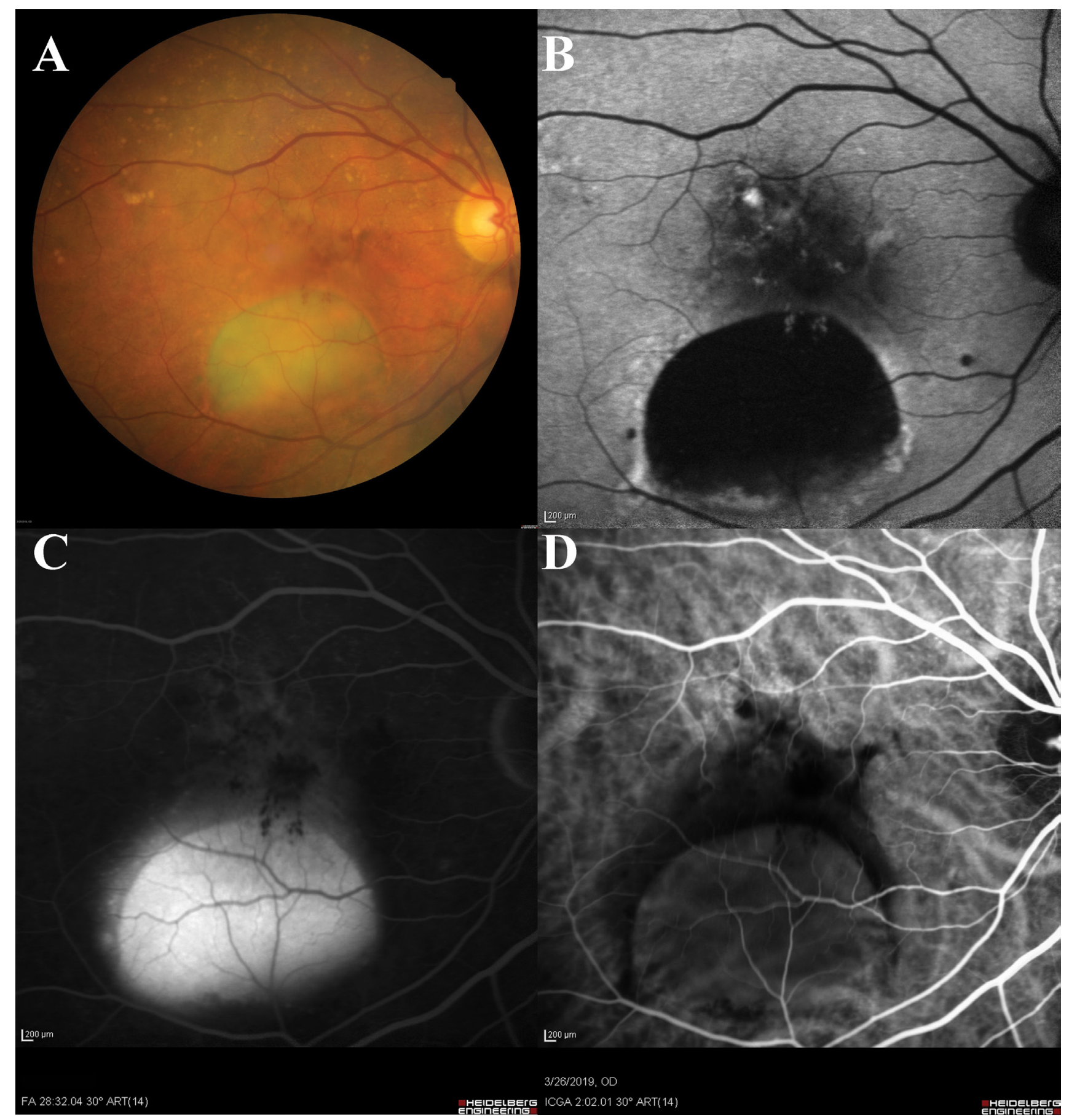

Figure 2: Multimodal imaging of retinal pigment epithelium rips (A) Colored fundus photo; (B) Fundus autofluorescence; (C) Fluorescein angiography; (D) Indocyanine green angiography of the right eye. 
Citation: Sunny CLA, Callie KLK (2020) Spontaneous Retinal Pigment Epithelium Rips under Fovea with Preserved Good Visual Acuity Outcome. J Healthcare 3(1):33-37

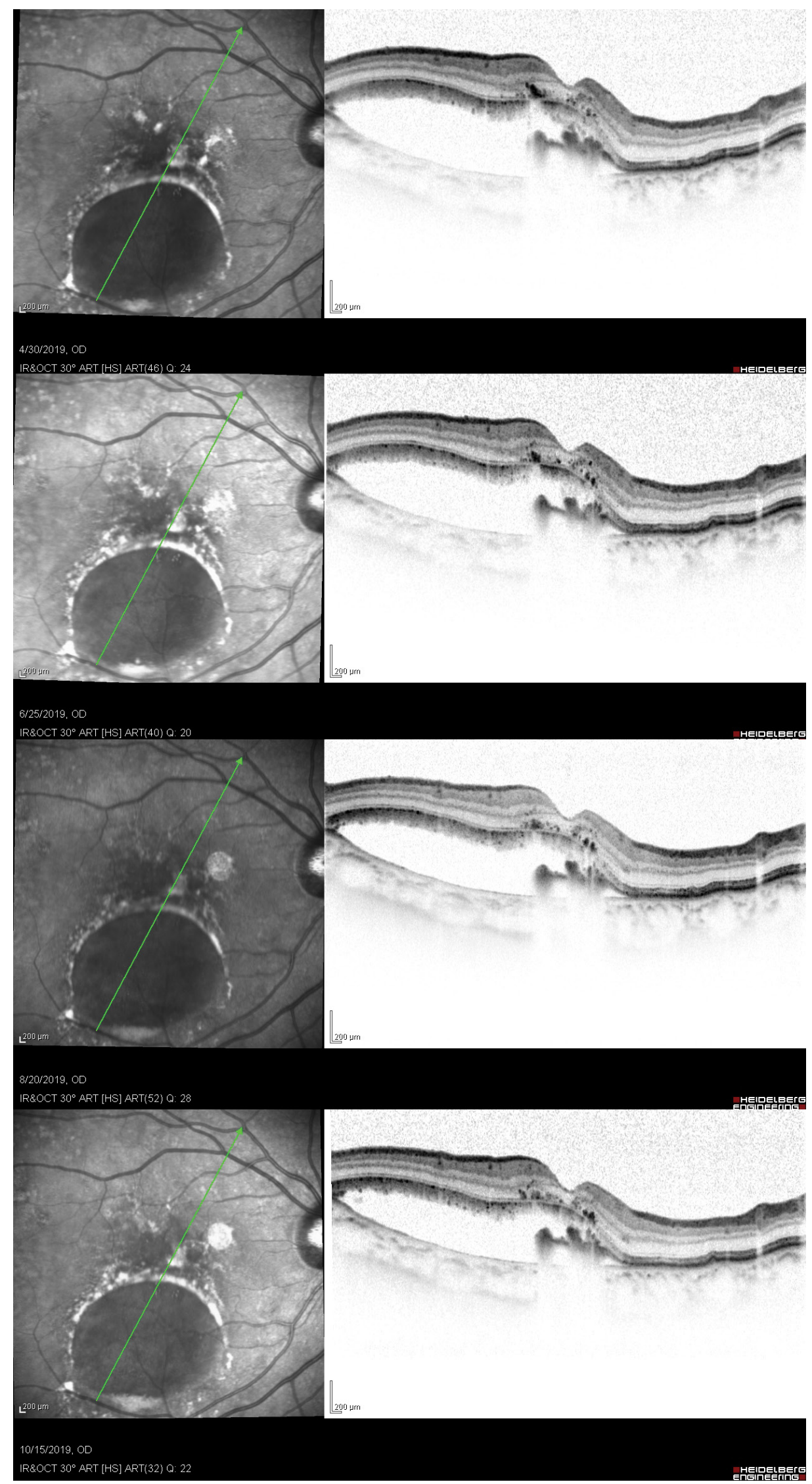

Figure 3: Follow up optical coherence tomography images for retinal pigment epithelium rips.

Morphology of the retinal pigment epithelium rips, preserved photoreceptor layer under the neurosensory retina and size of subretinal fluid were static on $1^{\text {st }}, 3^{\text {rd }}, 5^{\text {th }}, 7^{\text {th }}$ months after the rip. 


\section{References}

1. Sarraf D, Reddy S, Chiang A, et al. (2010) A new grading system for retinal pigment epithelial tears. Retina 30: 1039-1045.

2. Clemens CR, Eter N (2016) Retinal pigment epithelium tears: Risk factors, mechanism and therapeutic monitoring. Ophthalmologica 235: 1-9.

3. Ersoz MG, Karacorlu M, Arf S, (2017) Retinal pigment epithelium tears: Classification, pathogenesis, predictors, and management. Surv Ophthalmol 64: 493-505.
4. Caramoy A, Kirchhof B, Fauser S (2011) Retinal pigment epithelium tears secondary to age-related macular degeneration: A simultaneous confocal scanning laser ophthalmoscopy and spectral-domain optical coherence tomography study. Arch Ophthalmol 129: 575-579.

5. Giannakaki Zimmermann H, Querques G, Munch IC, et al. (2017) Atypical retinal pigment epithelial defects with retained photoreceptor layers: A so far disregarded finding in age related macular degeneration. BMC Ophthalmol 17: 67. 